\title{
Influencia de la hipoxia hipóxica sobre algunos parámetros hematológicos, cardiovasculares y respiratorios de varones que residen en el valle del Colca (3417 - 3633 msnm).
}

Influence of hypoxic hypoxia on some hematological, cardiovascular and respiratory parameters of men residing in the Colca Valley (3417-3633 msnm.)

\section{Edgard Quispe Alfaro y Henry Díaz-Murillo}

Universidad Nacional de San Agustín. Arequipa Perú.

\section{INFORMACIÓN}

\section{Historia del Artículo \\ Recepción: 28/04/2019 \\ Revisión: 21/06/2019}

Aceptación: 25/06/2019

\section{Palabras Clave}

Hipoxia, sangre, índice de masa corporal y respiración

\section{Key Words}

Hypoxia, blood, body mass index and breathing.

\section{DOI}

https://doi.org/10.35286/veritas. v20i2.249

\begin{abstract}
RESUMEN
Se evaluó la influencia de la hipoxia hipóxica sobre algunos parámetros hematológicos, cardiovasculares y respiratorios de varones que residen en el valle del Colca ( $3417-3633$ msnm). La evaluación experimental se realizó en el Centro de Salud de Chivay y Puesto de Salud de Yanque, durante los meses de enero a diciembre del 2015. El promedio de edad fue de 22.76 \pm 5.04 años, peso promedio es de $58.94 \pm 6.16 \mathrm{Kg}$, talla promedio de $1.64 \pm 5.02 \mathrm{mt}(\mathrm{p}>0.05)$. No se encontró diferencia significativa en la edad, peso, talla e IMC en los pobladores de Chivay, Yanque y Achoma. Se encontró diferencia significativa en el recuento eritrocitario, hematocrito $y$ hemoglobina en los pobladores de Chivay, Yanque y Achoma $(\mathrm{p}<0.05)$. No se encontró diferencia significativa en el recuento leucocitario en los pobladores evaluados $(p>0.05)$. No se encontró diferencia significativa en el recuento leucocitario en los pobladores de Chivay, Yanque y Achoma ( $>0.05)$. Se encontró diferencia significativa en el VCM $(p<0.01)$. No se encontró diferencia significativa en los parámetros corpusculares HCM, CHCM. En la prueba de postcomparación de Tukey se encontró un aumento significativo $(p<0.05)$ en el VCM de los individuos residentes de Chivay. Se encontró diferencia significativa en el pulso $(\mathrm{p}<0.05)$. No se encontró diferencia significativa en los parámetros corpusculares PS, PD, PP, PMF. En la prueba de postcomparación de Tukey se encontró un aumento significativo $(\mathrm{p}<0.05)$ en el pulso de los individuos residentes de Chivay. No se encontró diferencia significativa en el diámetro de tórax $\mathrm{y}$ frecuencia respiratoria $(\mathrm{p}>0.05)$.
\end{abstract}

\begin{abstract}
The influence of hypoxic hypoxia on some hematological, cardiovascular and respiratory parameters of men residing in the Colca Valley (3417-3633 masl) was evaluated. The experimental evaluation was carried out at the Chivay Health Center and Health Post of Yanque, during the months of January to December 2015. The average age was $22.76 \pm 5.04$ years, average weight is $58.94 \pm 6.16 \mathrm{Kg}$, average height of $1.64 \pm 5.02 \mathrm{mt}(\mathrm{p}>0.05)$. There was no significant difference in age, weight, height and BMI in the residents of Chivay, Yanque and Achoma. Significant difference in erythrocyte count, hematocrit and hemoglobin was found in the inhabitants of Chivay, Yanque and Achoma $(p<0.05)$. There was no significant difference in leukocyte count in the assessed population ( $p>0.05)$. There was no significant difference in leukocyte count in the inhabitants of Chivay, Yanque and Achoma ( $p>0.05$ ). A significant difference was found in the MCV $(\mathrm{p}<0.01)$. No significant difference was found in the corpuscular parameters HCM, CHCM. In the Tukey post-comparison test a significant increase $(\mathrm{p}<0.05)$ was found in the VCM of the Chivay resident individuals. A significant difference was found in the pulse (p $<0.05$ ). No significant difference was found in the PS, PD, PP, PMF corpuscular parameters. In the Tukey post-comparison test a significant increase $(p<0.05)$ was found in the pulse of Chivay resident individuals. No significant difference was found in the chest diameter and respiratory rate $(\mathrm{p}>0.05)$
\end{abstract}

\section{INTRODUCCIÓN}

En la región andina de nuestro país el residente nativo ha estado bajo la influencia de los factores ambientales de la altitud por milenios, pero sólo en los últimos años del siglo pasado se ha iniciado el estudio de la fisiología del hombre nativo y residente no nativo de altura. En los últimos decenios

Correspondencia:

Henry Díaz-Murillo

hdiazmur@gmail.com el desarrollo de la aviación ha estimulado extensamente la investigación de los efectos fisiológicos de la altura, a fin de definir los límites de exposición tolerable y segura, para desarrollar medidas protectoras más efectivas.

Un medio de gran altitud impone sobre el cuerpo humano ciertas dificultades y limitaciones en el mantenimiento y coordinación satisfactoria de sus procesos fisiológicos. En tal medio la densidad del aire está disminuida y la temperatura y humedad son bajas. Pero el principal factor y el más estudiado, es una condición de hipoxia o de deficiencia de oxígeno. Como consecuencia de la reducida presión barométrica, la presión parcial de oxígeno en el aire inspirado es baja, y bajo 
estas circunstancias la hemoglobina de la sangre que circula a través de los pulmones llega a ser menos saturada con este gas. Este hecho junto con la disminuida tensión de la fracción físicamente disuelta en el plasma hace más difícil la difusión y utilización de oxígeno a nivel tisular.

Los estudios de altura, son útiles para comprender mejor los problemas clínicos que se encuentran diariamente a nivel del mar a pesar de las diferencias obvias. Numerosos trabajos han sido publicados (Monge, Hurtado, Rotta, Velásquez, Marticorena, etc.) sobre los mecanismos fisiológicos y bioquímicos, que los habitantes de altura ponen en juego, a fin de adecuar el funcionamiento del organismo, a las condiciones ambientales existentes en esas regiones, lo que les permite vivir y trabajar en esos ambientes sin daño alguno para la salud. En contraposición a estos sujetos debidamente adaptados, se encuentran los que adolecen del denominado Mal de Montaña Crónico o Mal de Monge, descrito por este en 1928, que presenta una cuadro sintomático característico que desaparece cuando son trasladados a nivel del mar.

En el campo hematológico se ha considerado al individuo de altura como policitémico, cuya poliglobulia es constante y proporcional a la altura que vive, como lo demuestran Whittembury, J. y Monge,C, en 1972, en sus estudios sobre edad y hematocrito en personas residentes a diferentes alturas y establecen que: " en la altura, el hematocrito es una función directa de la edad y proporcional a la altura de residencia"; hallazgos confirmados por los estudios de Sime,F. (1973).

La función respiratoria ha sido otro de los aspectos más estudiados de la adaptación del hombre a la altura, en razón de la aclimatación a las atmósferas con menor presión barométrica, es así que Sime en sus observaciones sobre ventilación y edad en sujetos de altura, obtiene " una línea de regresión con pendiente negativa, que indica que todos los individuos involucionan hacia la hipoventilación; o sea que, la pérdida de la ventilación es una condición progresiva y norma del individuo que habita en altura".

Por lo anteriormente señalado hemos investigado la influencia de la hipoxia hipóxica sobre algunos parámetros hematológicos, cardiovasculares y respiratorios de varones que residen en el valle del Colca ( 3417 - $3633 \mathrm{msnm}$ ). va orientado a contribuir al estudio de " la bioquímica y fisiología del hombre andino" que reside en la Provincia de Caylloma, determinando el efecto de la hipoxia hipóxica sobre los parámetros hematológicos (GR, Hb, Ht, VCM; HCM y CCHM), cardiovasculares (PS, PD, PP, PMF) y respiratorios (FR y Diámetro toráxico), así como de establecer la asociación de dichos parámetros y determinar los patrones de normalidad sobre dichos parámetros en varones que residen en altura, entre los $3417-3633 \mathrm{msnm}$ y que de acuerdo a la clasificación de Monge,C corresponde a un lugar habitable que es la máxima altura en la cual el ser humano puede tolerar la hipóxia crónica sin menoscabo de sus funciones vitales.

\section{MATERIAL Y MÉTODOS \\ Unidades de estudio}

Durante el desarrollo del presente trabajo, se evaluaron a 105 varones cuya inclusión en el trabajo se consideró los siguientes criterios:

\section{Criterios de inclusión}

- Varones que hayan nacido y crecido en las zonas de estudio y que vivan hasta la actualidad

- Varones que hayan nacido en altitudes menores o a nivel del mar, pero que por diferentes razones residan en las zonas de estudio por más de un dos y sin interrupciones.

- Varones clínicamente sanos

- Varones mayores de 18 años y menores a 50 años de edad.

\section{Criterios de exclusión}

- Varones que residan menos de dos años

- Varones que no tengan antecedentes de ser fumadores, ni que hayan padecido enfermedades graves, sobre todo: TBC, neumonía, asma bronquial, diabetes, nefropatías, cardiopatías ni accidentes graves.

- De no haber sido sometidos a ningún tipo de intervenciones quirúrgicas durante su vida que hayan sido de riesgo.

- Descarto a varones de nacionalidad extranjera

\section{Localización}

El presente estudio se llevó a cabo en los distritos de Chivay, Yanque y Achoma de la provincia de Caylloma, departamento de Arequipa. Distritos ubicados a.

\begin{tabular}{cccc}
\hline DISTRITO & MSNM & LATITUD SUR & LATITUD OESTE \\
\hline CHIVAY & 3633 & $15038^{\prime} 02^{\prime \prime}$ & $71035^{\prime} 58^{\prime \prime}$ \\
YANQUE & 3417 & $15038^{\prime} 45^{\prime \prime}$ & $71039^{\prime} 24^{\prime \prime}$ \\
ACHOMA & 3450 & $15039^{\prime} 30^{\prime \prime}$ & $71035^{\prime} 58^{\prime \prime}$ \\
\hline
\end{tabular}

Y que cuentan con una población de:

\begin{tabular}{cccc}
\hline DISTRITO & CHIVAY & YANQUE & ACHOMA \\
\hline $\begin{array}{c}\text { Población Total } \\
\text { Hombres }\end{array}$ & 4051 & 2220 & 1304 \\
$\begin{array}{c}15-54 \text { años } \\
\text { Muestra }\end{array}$ & 938 & 353 & 287 \\
& 45 & 44 & 16 \\
\hline
\end{tabular}

Tomado de los datos estadísticos del Puesto de Salud de Yanque. 


\section{METODOLOGÍA}

El presente estudio de tipo prospectivo, de corte transversal y es de carácter descriptivo a analítico.

Basados los criterios de inclusión al presente estudio y contando con la valiosa colaboración del cuerpo médico y de enfermeras del Puesto de Salud de Yanque y Chivay se procedió al llenado de la ficha personal (Anexo 1) en el que se consideran la edad, procedencia, tiempo de residencia, actividad diaria, evaluaciones físicas como el diámetro toráxico antero posterior, peso, estatura, frecuencia respiratoria, frecuencia cardiaca, presión diastólica, presión sistólica y pulso.

Teniendo una opinión favorable del médico se procedió a la toma de una muestra sanguínea al total de varones en estudio, en horas de la mañana previo ayuno, en forma aséptica y con material descartable. Tomando aproximadamente $5 \mathrm{cc}$ de sangre de la vena localizada en la fosa antecubital, en un frasco heparinizado (5 ul).

\section{Determinación de la hemoglobina $(\mathrm{Hb})$}

\section{Método}

Cianometahemoglobina

\section{Fundamento del Método}

El ión ferroso $(\mathrm{Fe}++)$ de la molécula hem de la hemoglobina se oxida a hierro férrico $(\mathrm{Fe}+++)$ por el ferricianuro dando lugar a metahemoglobina (Met-Hb) que es a continuación transformada en cianometaheglobina por el cianuro de potasio la que es de color rojo. Se mide a $540 \mathrm{~nm}$.

\section{Equipo}

Espectofotómetro (photometer 4010 Clinicon Mannheim GMBH

Pipeta Sahlii

Solución Drabkin:

- Bicarbonato de sodio

- Cianuro de potasio$$
1.0 \mathrm{~g}
$$

- Ferricianuro de potasio

- Agua destilada

Condiciones de reacción:

Longitud de onda $=540 \mathrm{~nm}$

Tiempo de reacción $=5 \mathrm{~min}$

Volumen de solución Drabkin $=5 \mathrm{ml}$

Volumen de muestra $($ Sangre total $)=0.02 \mathrm{ml}$

Volumen final de reacción $=5.02 \mathrm{ml}$

\section{Procedimiento}

En un tubo de ensayo se coloca $5.0 \mathrm{ml}$ de solución Drabkin, luego se mide $0.02 \mathrm{ml}$ de muestra de sangre con la pipeta de Sahlii, se agita bien y se espera 5 min para leer la absorbancia de la muestra a $540 \mathrm{~nm}$

El estándar utilizado fue del laboratorio Wiener, el cual presenta una concentración de $18 \mathrm{~g} \%$ y se prepara con $50 \mathrm{ml}$ de solución Drabkin. Con esta solución se lee la absorbancia a $540 \mathrm{~nm}$.
Cálculo de los resultados:

Hg g \% = Absorbancia de la Muestra X Factor

$$
\begin{aligned}
& \text { factor }=\frac{S t}{\text { Absorbantadip } / S t} \\
& \text { Factior }=\frac{18 \mathrm{~g} \%}{0.4737}=38 \mathrm{~g} \%
\end{aligned}
$$

\section{Determinación de hematocrito (Ht)}

Determinación del volumen de masa eritrocitaria expresada en porcentaje.

\section{Método}

Técnica del microhematocrito, se fundamenta en el uso de tubos capilares heparinizados de $7 \mathrm{~cm}$ de largo por $1 \mathrm{~mm}$ de diámetro interior. Técnica rápida que consiste en separar los eritrocitos del plasma por centrifugación, obteniéndose hematíes aglomerados, los cuales son medidos en la escala para microhemtocrito (ABACO-GELEC).

\section{Equipo}

Centrifuga Gelec-G12 automatizada de 12000 revoluciones

\section{Procedimiento}

Se llena el tubo capilar heparinizado con la muestra de sangre hasta las $3 / 4$ partes de la extensión total del capilar por capilaridad, luego se sella uno de los extremos con plastilina. Después se coloca el capilar en la centrifuga a una velocidad de 10000 r.p.m. por 5 minutos.

\section{Lectura}

Se lee el tanto por ciento del volumen de las células sanguíneas directamente en la escala especial (ABACOGELEC), colocando el tubo capilar de manera tal que el nivel superior de la plastilina coincida con la línea $0 \%$ y el nivel superior con la línea $100 \%$. Esto se logra deslizando el capilar de izquierda a derecha. Se determina el valor del hematocrito efectuando la lectura que corresponde a la zona de separación plasma-glóbulos rojos.

\section{Estudio numérico de los elementos formes de la sangre}

\section{Recuento de eritrocitos o glóbulos rojos}

Determinación del número de eritrocitos en milímetro cúbico o por litro de sangre

\section{Método}

Se fundamenta el uso de la pipeta de Thoma para glóbulos rojos, la cual viene calibrada para diluir la muestra de sangre en dilución de 1 en 200 ó 1 en 20, para ello se utiliza la solución diluyente de Hayem que tiene la propiedad de lisar los leucocitos. Permitiendo una mayor visibilidad de los eritrocitos. 


\section{Materiales e instrumentos}

Microscopio óptico compuesto

Contador manual

Hemocitómeetro, se utilizó la cámara de Neubawer

Pipeta de Thoma, con su respectiva sonda de absorción

Solución de Hayem

Cloruro de mercurio

Sulfato sódico

Cloruro sódico

Agua destilada

$$
\begin{gathered}
0.5 \mathrm{~g} \\
5.0 \mathrm{~g} \\
1.0 \mathrm{~g} \\
200.0 \mathrm{ml}
\end{gathered}
$$

\section{Procedimiento técnico}

Se aspira sangre exactamente hasta la señal $0.5 \mathrm{ml}$ de la pipeta de dilución, luego se aspira la solución de Hayem hasta la señal 101 del bulbo. Se agita cuidadosamente durante 2 ó 3 min para asegurar una mezcla total entre la sangre y el diluyente.

Para el llenado de la cámara de Neubawer se coloca el cubre objetos sobre la zona cuadriculada del hemocitómetro. Se agita la pipeta, se desechan las 3 ó 4 primeras gotas de sangre diluida y luego con cuidado se llena la cámara por capilaridad, colocando la punta de la pipeta en uno de los extremos del cubre objetos. Se deja que la mezcla sedimente en la cámara para después realizar el conteo de células con el objetivo de inmersión (100 X).

Los conteos se realizaron por duplicado.

\section{Cálculo de los resultados}

La forma básica para el cálculo de número de células sanguíneas por milímetro cúbico de sangre no diluida es la siguiente.

\section{NrodeGrpormm ${ }^{3}=$ NoodeGricontados $\times 10000$}

Donde:

GR $=$ Glóbulos Rojos

Nro $=$ Número de glóbulos rojos contados

Esta fórmula se basa en lo siguiente:

Nro de GR por mm3 = Nro de GR contados en 5 cuadrados Area Contada $(\mathrm{mm} 2)$ x Altura de la Cámara $(\mathrm{mm})$ x Dilución

Nro de GR por mm3 = Células contadas

$$
1 / 5 \times 1 / 10 \times 1 / 200
$$

\section{Recuento de leucocitos o glóbulos blancos}

Determinación del número de luecocitos por milímetro cúbico o por litro de sangre.

\section{Método}

Pipeta de dilución de luecocitos, se fundamenta en el uso de la pipeta de Thoma para glóbulos blancos, la cual viene calibrada para diluir la muestra de sangre en 1 en 20. Para ello se utiliza la solución diluyente de Turk que tiene la propiedad de lisar a los eritrocitos. Permitiendo una mayor visibilidad de los leucocitos.

\section{Material e instrumentos}

Microscopio óptico compuesto

Cámara de neubawer

Contador manual

Pipeta de Thoma para glóbulos blancos

Solución de Turk

Ácido acético glacial $\quad 1.0 \mathrm{ml}$

Solución acuosa de Violeta de Genciana al 1\% $\quad 2.5 \mathrm{ml}$

Agua destilada $\quad 100 \mathrm{ml}$

\section{Procedimiento}

Aspiramos la sangre hasta la marca de 0.5 , luego se completa con el diluyente (solución de Turk) hasta la marca 11 después se agita por 2 ó 3 minutos y se deja en reposo por 10 minutos.

Se carga la cámara de Neubawer previamente preparada como en el caso del recuento de los glóbulos rojos, desechando antes 2 ó 3 gotas. Se deja reposar por 3 minutos para que los leucocitos se depositen en el retículo de la cámara. Se realiza el recuento con el objetivo de $40 \mathrm{X}$

\section{Cálculo de los resultados}

Los resultados se dan contando el número de leucocitos en las 4 cuadrículas grandes y extremas de la cámara multiplicando la suma total por 50 (dilución $1 / 20$ ), esto se explica con el cálculo siguiente:

Nro de GB/ $\mathrm{mm}=\mathrm{GB}$ contados en las cuatro cuadrículas Área contada $(\mathrm{mm} 2)$ x altura de la cámara $(\mathrm{mm})$ x dilución de sangre

Nro de GB $/ \mathrm{mm}=$ GB contados

$4 \times 1 / 10 \times 1 / 20$

Entonces: El Nro de leucocitos por mm3 = Nro de leucocitos contados x 50

\section{Índices corpusculares eritrocitarias (constantes hemáticas)}

Sobre la base del conteo de los eritrocitos y los valores de $\mathrm{Hb}$ y Ht, se puede formular las características de los hematíes de un individuo, los que en éste caso ayudarán al diagnóstico del efecto de la altura.

\section{Volumen corpuscular medio (VCM)}

El VCM es el volumen medio de los hematíes de una muestra de sangre. Se calcula

$$
\begin{aligned}
\mathrm{VCM}(\mathrm{ul})= & \mathrm{Ht} \% \times 10 \\
& \text { Millones de GR por mm3 entre } 10(6)
\end{aligned}
$$

Valores normales: entre $85-95 \mathrm{ul}$

\section{Hemoglobina corpuscular media (HCM)}

La HCM es la cantidad de Hb contenida en cada hemtíe. Se calcula

$$
\begin{aligned}
\mathrm{HCM}(\mathrm{pg})= & \mathrm{Hb} \mathrm{g} \% \mathrm{x} 10 \\
& \text { Millones de GR por mm3(6) }
\end{aligned}
$$

Valores normales: entre $30-34 \mathrm{pg}$ 


\section{Concentración corpuscular media de hemoglobina (CCMH)}

La CCMH expresa la concentración de $\mathrm{Hb}$ en cada hematíe. Se calcula

$$
\begin{aligned}
\mathrm{CCMH}(\mathrm{g} / \mathrm{l})= & \mathrm{Hb} \mathrm{g} \% \times 100 \\
& \mathrm{Ht} \%
\end{aligned}
$$

Valores normales: entre $32-34 \%$

\section{Medida de la presión arterial por el método auscultatorio (KOROTKOFF)}

\section{Materiales}

- Esfigmomanómetro.

- Estetoscopio.

- Reloj con segundero

\section{Procedimiento}

1. El paciente deberá sentarse cómodamente, colocando el brazo sobre la mesa de modo que quede a nivel del corazón.

2. Se ubica por palpitación el pulso de la arteria cubital, por encima del pliegue del codo.

3. Se coloca el brazalete de Riva Rossi, completamente desinflamado, alrededor del brazo de manera que su borde inferior quede $2 \mathrm{~cm}$ sobre el pliegue del codo, y que la bolsa de goma quede justamente sobre la arteria braquial.

4. Se coloca el diafragma del estetoscopio exactamente sobre la arteria braquial sin tocar el manguito.

5. Se sube la presión del manómetro por encima de $30 \mathrm{~mm}$ $\mathrm{Hg}$ después de que no haya pulso radial

6. Se abre la válvula lentamente de modo que la presión del manómetro descienda 2-3 $\mathrm{mm} \mathrm{Hg}$ por segundo. $\mathrm{Al}$ escuchar el primer ruido sincrónico se debe anotar la presión del manómetro; este momento corresponde a la presión sistólica. A medida que sigue bajando la presión, los sonidos cambiaran de intensidad y de calidad. Cuando los ruidos desaparecen o se hacen más débiles y apagados, anote el valor de la presión la que corresponderá a la presión diastólica.

\section{Evaluación respiratoria}

El observador deberá primero determinar la frecuencia respiratoria por minuto mientras el sujeto está sentado o en estado de reposo. El aire exhalado puede fácilmente ser detectado a medida que fluye sobre el dedo humedecido. El dedo se enfriará a medida que el aire produce evaporación del agua. No se comienza a contar sino después de varias exhalaciones, de tal forma que el sujeto tendrá tiempo de ajustarse a las condiciones de la prueba y podrá respirar normalmente.

\section{Análisis estadístico}

Para el análisis e interpretación de los resultados obtenidos, se aplicó el paquete estadístico SPSS 21 para Windows 10.

\section{RESULTADOS}

El presente trabajo de tesis, se realizó en el laboratorio de Fisiología Animal de la Escuela Profesional de Biología de la UNSA y en los laboratorios del Centro de Salud de Chivay y Puesto de Salud de Yanque.

Tabla 1: Parámetros antropométricos de varones que

\begin{tabular}{|c|c|c|c|c|c|c|c|}
\hline PARÁMETRO & $\begin{array}{l}\text { YANQUE } \\
(44)\end{array}$ & $\begin{array}{c}\text { ACHOMA } \\
\text { (16) }\end{array}$ & $\begin{array}{c}\text { CHIVAY } \\
(45)\end{array}$ & PROM & $\mathrm{F}$ & $\mathrm{P}$ & S \\
\hline EDAD & $\begin{array}{c}23.23 \pm \\
5.31\end{array}$ & $22.18 \pm 4.9$ & $\begin{array}{c}22.51 \pm \\
4.89\end{array}$ & $\begin{array}{c}22.76 \pm \\
5.04\end{array}$ & 0.34 & 0.71 & NS \\
\hline PESO & $\begin{array}{c}58.63 \pm \\
6.33\end{array}$ & $58,96 \pm 6.74$ & $\begin{array}{c}59.24 \pm \\
5.90\end{array}$ & $\begin{array}{c}58.94 \pm \\
6.16\end{array}$ & 0.11 & 0.89 & NS \\
\hline ESTATURA & $\begin{array}{c}1.64 \pm \\
4.47 \times 10-2\end{array}$ & $\begin{array}{c}1.63 \pm \\
3.89 \times 10-2\end{array}$ & $\begin{array}{c}1.64 \pm \\
5.89 \times 10-2\end{array}$ & $\begin{array}{l}1.64 \pm \\
5.02\end{array}$ & 0.15 & 0.86 & NS \\
\hline IMC & $\begin{array}{c}21.78 \pm \\
1.78\end{array}$ & $22.05 \pm 2.07$ & $\begin{array}{c}21.96 \pm \\
1.45\end{array}$ & & 0.23 & 0.79 & NS \\
\hline
\end{tabular}
residen en el valle del Colca ( 3417 - 3633 MSNM)

En la Tabla y Gráfico 1 se observó un promedio de edad de $22.76 \pm 5.04$ años, cuyo rango oscila entre los 16 y 40 años, en cuanto al peso promedio es de $58.94 \pm 6.16$ cuyo rango oscila entre 45.8 y $73 \mathrm{Kg}$. en lo referente a la estatura se encontró un promedio de $1.64 \pm 5.02 \mathrm{mts}$. No se encontró diferencia significativa en la edad, peso, talla e IMC en los pobladores de Chivay, Yanque y Achoma.

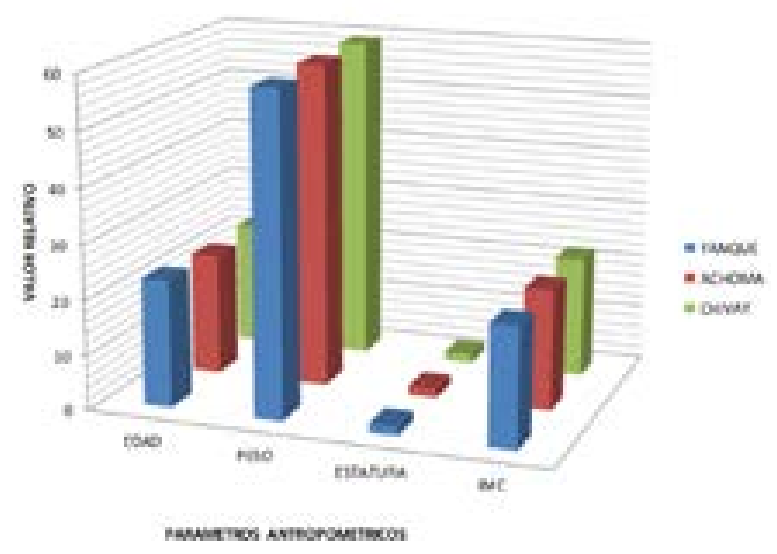

Gráfico 1: Parámetros antropométricos de varones que residen en el valle del Colca (3417 - 3633 MSNM) 
Tabla 2: Parámetros eritrocitarios de varones que residen en el valle del Colca (3417 - 3633 MSNM)

\begin{tabular}{ccccc}
\hline ZONA & $\mathrm{N}$ & $\mathrm{RE}(\mathrm{X} 106)$ & $\mathrm{Ht}(\%)$ & $\mathrm{Hb}(\mathrm{g} \%)$ \\
\hline YANQUE & 44 & $5.54 \pm 0.30 \mathrm{a}$ & $51.55 \pm 3.65 \mathrm{a}$ & $16.94 \pm 1.17 \mathrm{a}$ \\
ACHOMA & 16 & $5.61 \pm 0.16 \mathrm{ab}$ & $53.50 \pm 0.71 \mathrm{ab}$ & $17.37 \pm 0.71 \mathrm{ab}$ \\
CHIVAY & 45 & $5.67 \pm 0.17 \mathrm{~b}$ & $54.44 \pm 3.19 \mathrm{~b}$ & $17.79 \pm 1.01 \mathrm{~b}$ \\
$\begin{array}{c}\text { TOTAL } \\
\text { Prueba } \\
\text { de F }\end{array}$ & $3.948 \mathrm{p} \pm 0.05)$ & $8.66(\mathrm{p} \pm 0.01)$ & $7.438 \mathrm{p} \pm 0.01)$ \\
P & $0.02 \mathrm{x}$ & $0.0003 \mathrm{xx}$ & $0.0009 \mathrm{xx}$ \\
\hline
\end{tabular}

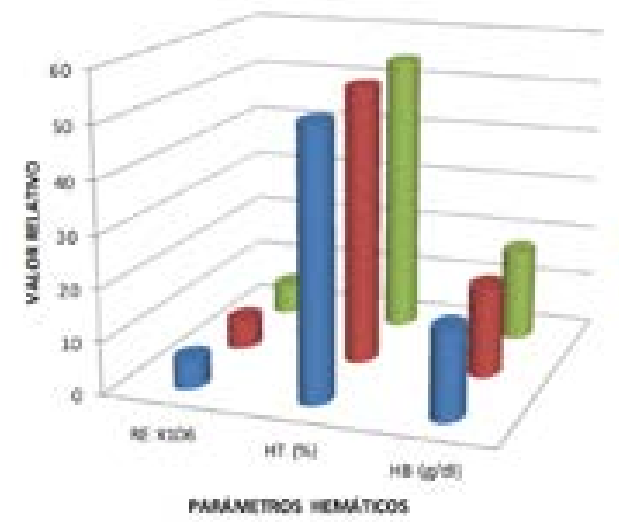

- vavocar

- noverar

sorvir

Gráfico 2: Parámetros eritrocitarios de varones que residen en el valle del Colca (3417 -3633 MSNMC

Tabla 3: Recuento leucocitario de varones que residen en el valle del Colca (3417 - 3633 MSNM)

\begin{tabular}{ccc}
\hline ZONA & $\mathrm{N}$ & $\mathrm{RL}(\mathrm{mm} 3)$ \\
\hline YANQUE & 44 & $7227.27 \pm 457.28 \mathrm{a}$ \\
ACHOMA & 16 & $7043.75 \pm 117.83 \mathrm{a}$ \\
CHIVAY & 45 & $7096.67 \pm 414.43 \mathrm{a}$ \\
TOTAL & 105 & $7143.33 \pm 411.44$ \\
Prueba de F & & $1.70(\mathrm{p} \pm 0.05)$ \\
P & & 0.19 \\
\hline
\end{tabular}

En la Tabla y Gráfico 3 No se encontró diferencia significativa en el recuento leucocitario en los pobladores de Chivay, Yanque y Achoma ( $\mathrm{p}>0.05)$.

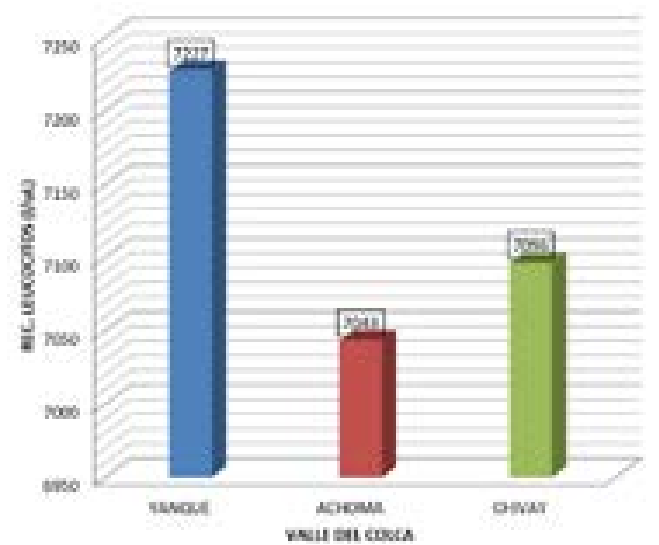

Gráfico 3: recuento leucocitario de varones que residen en el valle de la Colca (3417 -3633 MSNM)
Tabla 4: Índices hemáticos de varones que residen en el valle del Colca (3417 - 3633 MSNM)

\begin{tabular}{ccccc}
\hline ZONA & $\mathrm{N}$ & $\mathrm{VCM}(\mathrm{ul})$ & $\mathrm{HCM}(\mathrm{pg})$ & $\mathrm{CCHM}(\mathrm{g} \%$ \\
\hline YANQUE & 44 & $93.11 \pm 3.32 \mathrm{a}$ & $30.60 \pm 1.05 \mathrm{a}$ & $32.87 \pm 0.39 \mathrm{a}$ \\
& & & & \\
ACHOMA & 16 & $95.43 \pm 5.63 \mathrm{ab}$ & $30.96 \pm 1.12 \mathrm{ab}$ & $32.50 \pm 1.37 \mathrm{a}$ \\
& & & & \\
CHIVAY & 45 & $95 . .6 \pm 3.69 \mathrm{a}$ & $31.37 \pm 1.22 \mathrm{~b}$ & $32.69 \pm 0.34 \mathrm{~b}$ \\
TOTAL & 105 & $94.69 \pm 4.09$ & $30.98 \pm 1.18$ & $32.74 \pm 0.67$ \\
Prueba de F & & $6.31(\mathrm{p} \pm 0.01)$ & $5.05(\mathrm{p} \pm 0.01)$ & $2.22(\mathrm{p} \pm 0.05)$ \\
P & & $0.0025 \pm 0.01$ & $0.081 \pm 0.05$ & $0.11 \pm 0.05$ \\
S & & AS & NS & $\mathrm{NS}$ \\
\hline
\end{tabular}

En la Tabla y Gráfico 4 se encontró diferencia significativa en el VCM $(\mathrm{p}<0.01)$. No se encontró diferencia significativa en los parámetros corpusculares HCM, CHCM. En la prueba de postcomparación de Tukey se encontró un aumento significativo $(\mathrm{p}<0.05)$ en el VCM de los individuos residentes de Chivay.

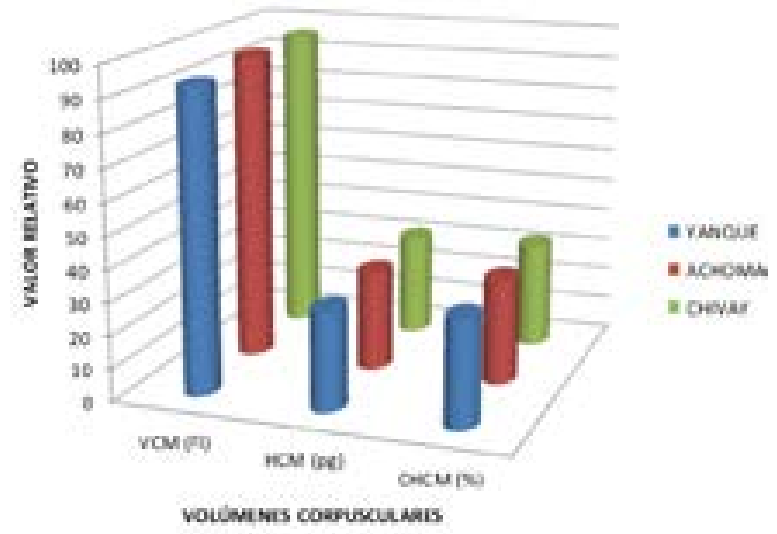

Gráfico 4: Índices hemáticos de varones que residen en el valle del Colca (3417 - 3633 MSNM)

Tabla 5: Parámetros cardiovasculares de varones que residen en el valle del Colca (3417 - 3633 msnm)

\begin{tabular}{|c|c|c|c|c|c|c|}
\hline ZONA & $\mathrm{N}$ & PS & PD & PULSO & PP & PMF \\
\hline YANQUE & 44 & $\begin{array}{c}110.46 \pm 8.88 \\
\mathrm{a}\end{array}$ & $70.23 \pm 5.90 \mathrm{a}$ & $\begin{array}{c}75.27 \pm 7.85 \\
\mathrm{a}\end{array}$ & $\begin{array}{c}39.55 \pm 9.87 \\
\mathrm{a}\end{array}$ & $\begin{array}{c}83.41 \pm 5.50 \\
\mathrm{a}\end{array}$ \\
\hline ACHOMA & 16 & $\begin{array}{c}114.38 \pm 10.94 \\
\mathrm{a}\end{array}$ & $\begin{array}{c}68.75 \pm \\
6.19 \mathrm{a}\end{array}$ & $\begin{array}{c}74.88 \pm 5.12 \\
\mathrm{a}\end{array}$ & $\begin{array}{c}46.88 \pm 10.78 \\
\mathrm{~b}\end{array}$ & $\begin{array}{cc}8 & 84.38 \\
& \pm 6.64 \mathrm{a}\end{array}$ \\
\hline CHIVAY & 45 & $\begin{array}{c}110.44 \pm 8.52 \\
\mathrm{a}\end{array}$ & $\begin{array}{c}69.11 \pm \\
5.96 \mathrm{a}\end{array}$ & $\begin{array}{c}76.73 \pm 5.35 \\
\mathrm{a}\end{array}$ & $\begin{array}{c}41.11 \pm 10.05 \\
a b\end{array}$ & $\begin{array}{c}582.82 \pm 5.12 \\
\mathrm{a}\end{array}$ \\
\hline TOTAL & 105 & $111.05 \pm 9.06$ & $69.52 \pm 5.95$ & $75.84 \pm 6.48$ & $41.33 \pm 10.29$ & $983.30 \pm 5.50$ \\
\hline Prueba de F & & $\begin{array}{c}1.27( \\
\mathrm{p} \pm 0.05)\end{array}$ & $0.55(\mathrm{p} \pm 0.05)$ & $\begin{array}{c}0.77( \\
\mathrm{p} \pm 0.05)\end{array}$ & $\begin{array}{c}3.12( \\
\mathrm{p} \pm 0.05)\end{array}$ & $\begin{array}{c}0.49( \\
\mathrm{p} \pm 0.05)\end{array}$ \\
\hline $\mathrm{P}$ & & $0.28 \pm 0.05$ & $0.58 \pm 0.05$ & $0.47 \pm 0.05$ & $0.05 \pm 0.05$ & $0.62 \pm 0.05$ \\
\hline S & & NS & NS & S & NS & NS \\
\hline
\end{tabular}


En la Tabla y Gráfico 5 se encontró diferencia significativa en el pulso $(p<0.05)$. No se encontró diferencia significativa en los parámetros corpusculares PS, PD, PP, PMF. En la prueba de postcomparación de Tukey se encontró un aumento significativo $(\mathrm{p}<0.05)$ en el pulso de los individuos residentes de Chivay.

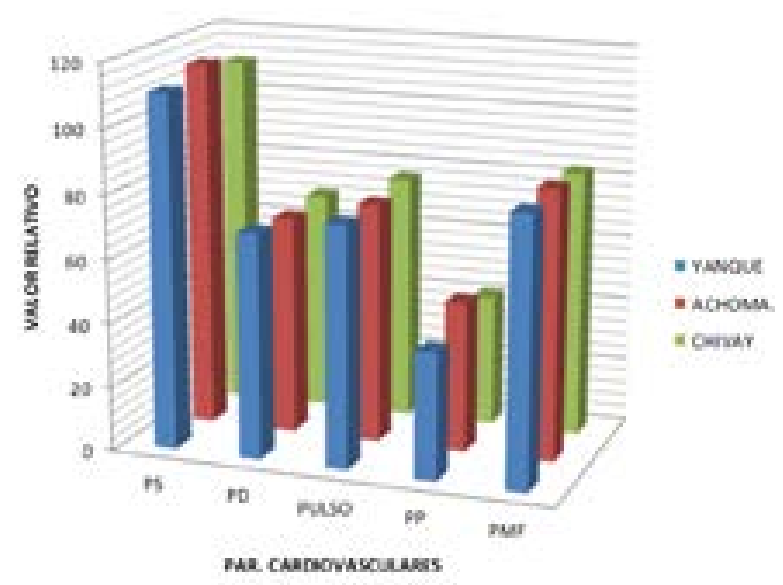

Gráfico 5: Parámetros cardiovasculares de varones que residen en el valle del Colca (3417-3633 MSNM)

Tabla 6: parámetros respiratorios de varones que residen en el valle del Colca (3417 - 3633 MSNM)

\begin{tabular}{cccc}
\hline ZONA & $\mathrm{N}$ & $\begin{array}{c}\text { DIAMETRO } \\
\text { TORAX }(\mathrm{cm})\end{array}$ & FR RESPI \\
\hline YANQUE & 44 & $21.97 \pm 1.62 \mathrm{a}$ & $22.27 \pm 2.84 \mathrm{a}$ \\
ACHOMA & 16 & $21.78 \pm 1.62 \mathrm{a}$ & $22.81 \pm 2.07 \mathrm{a}$ \\
CHIVAY & 45 & $22.17 \pm 1.53 \mathrm{a}$ & $22.76 \pm 2.35 \mathrm{a}$ \\
TOTAL & 105 & $22.02 \pm 1.53$ & $22.56 \pm 2.35$ \\
Prueba de F & & $0.43(\mathrm{p} \pm 0.05)$ & $0.57(\mathrm{p} \pm 0.05)$ \\
P & & $0.65 \pm 0.05$ & $0.57 \pm 0.05$ \\
$\mathrm{~N}$ & & $\mathrm{NS}$ & $\mathrm{NS}$ \\
\hline
\end{tabular}

En la Tabla y Gráfico 6 no se encontró diferencia significativa en el diámetro de tórax y frecuencia respiratoria $(\mathrm{p}>0.05)$.

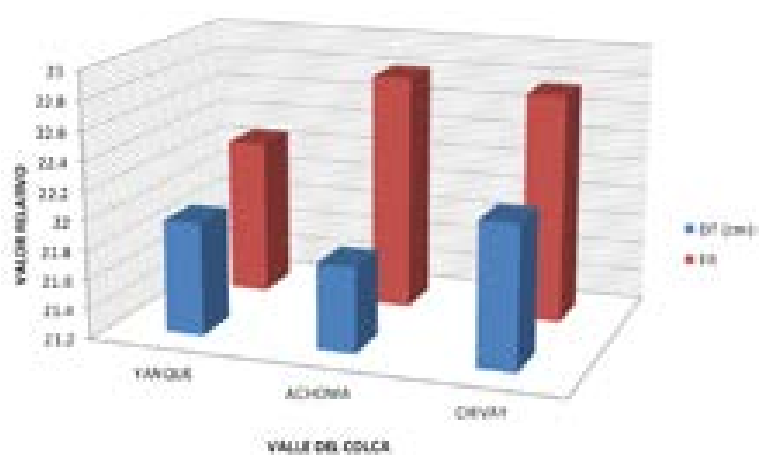

Gráfico 6: Parámetros respiratorios de varones que residen en el valle del Colca (3417 -3633 MSNM)

\section{DISCUSIÓN}

Se evaluó la influencia de la hipoxia hipóxica sobre algunos parámetros hematológicos, cardiovasculares y respiratorios de varones que residen en el valle del Colca (3417-3633 msnm).

Se observó un promedio de edad de $22.76 \pm 5.04$ años, cuyo rango oscila entre los 16 y 40 años, en cuanto al peso promedio es de $58.94 \pm 6.16$ cuyo rango oscila entre 45.8 y 73 $\mathrm{Kg}$. en lo referente a la estatura se encontró un promedio de $1.64 \pm 5.02 \mathrm{mts}$. No se encontró diferencia significativa en la edad, peso, talla e IMC en los pobladores de Chivay, Yanque y Achoma. La población evaluada es adulta con 18 años promedio de residencia en las zonas evaluadas, de forma que están plenamente climatizadas a la altura de Chivay (3633 m), Yanque (3417 m) y Achoma (3450 m). Aproximadamente 13 millones de personas viven permanentemente en situaciones de hipoxia en altitudes entre 3000-5000 metros sobre el nivel del mar. En estas altitudes la presión parcial del oxígeno es más baja y se corresponde con una concentración de oxígeno del $14-11 \%$ en condiciones normobáricas (en el nivel del mar). El hecho de que una población tan grande pueda residir permanentemente en esta altitud demuestra que la fisiología humana normal puede mantener la homeostasis en estas altitudes sin daño perceptible para la salud. La Alta Altitud (AA) se define a las alturas comprendidas entre 1800 y $6000 \mathrm{~m}$. Por encima de los 6000m (354 mm de Hg, correspondiendo a 9,5\% de oxígeno) denominamos Altitudes Extremas (AE). En general, la aclimatación humana a estos niveles de hipoxia que existen en Altitudes Extremas es imposible sin que se produzcan repercusiones negativas sobre la salud. Las alturas por debajo de $1800 \mathrm{~m}(610 \mathrm{~mm}$ $\mathrm{Hg}$, correspondiendo a concentraciones de $\mathrm{O} 2$ superiores al $17 \%$ ) no dan lugar a respuestas compensatorias y se pueden denominar Baja Altitud (BA). Además, la respiración intermitente con aire hipóxico y aire ambiente ha demostrado estimular la producción de antioxidantes en el cuerpo. Esta capacidad aumentada de los sistemas antioxidantes puede ayudar a eliminar desequilibrios tales como la formación de los radicales libres de oxígeno que favorecen la fatiga muscular en el ejercicio intenso y la alteración de las membranas celulares que están en el origen de un mayor riesgo de lesión Reeves y col (1985), Benziger y col (2015), Kiyamu y col (2015). El interés de los fisiólogos en el uso práctico de la aclimatación a la Alta Altitud, demostró que es un medio potente de estímulo de varios sistemas humanos. En respuesta a una disminución temporal de la concentración de oxígeno (hipoxia) nuestro cuerpo produce un número de modificaciones compensatorias. La estimulación de los mecanismos de defensa da lugar a los siguientes ajustes fisiológicos De la cruz y col (2014).

Se observó diferencia significativa en el recuento eritrocitario, hematocrito y hemoglobina en los pobladores de Chivay, Yanque y Achoma ( $>0.05)$. No se encontró diferencia significativa en el recuento leucocitario en los pobladores evaluados $(p>0.05)$. Pero estos valores se encuentran por encima de los valores normales a nivel del mar Gonzales (2003), Barbieri y col (2014). Este incremento se debe a la relación entre altitud, desarrollo y mantenimiento de la vida en los humanos, se puede inferir a partir del dato que señala que asentamientos poblacionales humanos, permanentes, no pueden existir más allá de una altitud de 5.300 m.s.n.m. La razón está en la relación que existe entre 
los valores de la $\mathrm{PB}$ y la presión que cada gas atmosférico tiene en cada valor de PB. Aun cuando el porcentaje relativo para cada gas se mantiene constante en la mezcla atmosférica, el número absoluto de sus moléculas disminuye a medida que crece la altura sobre el nivel del mar, así por ejemplo a 400 $\mathrm{m}$ el número total de moléculas de $\mathrm{O} 2$ ha disminuido en un 40\%. De los gases atmosféricos, el O2 es vital e insustituible para el desarrollo y mantenimiento de procesos orgánicos relacionados principalmente con la obtención de energía metabólica. Desde el aire atmosférico hasta su destino final en la mitocondria, el $\mathrm{O} 2$ se transporta mediante el proceso de difusión simple, la fuente de energía que permite esta difusión radica en el gradiente de concentración y/o de presión del gas, inspirado y su destino final en la mitocondria. Por lo tanto, datos de $\mathrm{g} / \mathrm{dl}$ de $\mathrm{Hb}$ y valores de \% Hct, deben siempre tomarse con precaución, mucho más en el caso que nos atañe, de presentar hechos y/o procesos de adaptación a la hipoxia altitudinal, la cual, como ya se ha mencionado impacta en forma directa a variables sanguíneas relacionadas con "almacenamiento" y entrega de O2 a los tejidos De ferrari y Col (2014).. Los estudios de Vargas y Col (2006), presentan resultados importantes en las medidas de la concentración de hemoglobina y en el valor del hematocrito para nativos de los Andes y del Tíbet que residen a más de 3.400 m.s.n.m. Los andinos presentan valores promedios de $3.5 \mathrm{~g} / \mathrm{dl}$ de $\mathrm{Hb}$ más que los tibetanos, incluso estos últimos pueden no presentar diferencias significativas con personas del nivel del mar. Se postula entonces que el mayor tiempo de permanencia de lo tibetanos en la altura ha permitido el desarrollo de adaptaciones genéticas que generan respuestas orgánicas a la hipoxia altitudinal, no relacionadas directamente con las variables sanguíneas tradicionales. Los trabajos de Pawson y $\mathrm{Col}$ (2010) realizados en residentes de gran altura, (3.400 m) el de Bighan y Col (2008), efectuado en altitud intermedia, $2.600 \mathrm{~m}$, presentan resultados contrarios. Es claro que en un tiempo tan corto de exposición a la hipoxia altitudinal no se pudo haber desarrollado una actividad eritropoyética que explique el incremento medido de un $9 \%$ en el conteo de eritrocitos. En el año 2004 Lingarde y col corrobora que a 2.600 m.s.n.m., el volumen de plasma en hombres residentes deportistas es un $8 \%$ menor que un grupo similar de nivel del mar, Brutsaert y col (2003), encuentra que también en mujeres se presenta esta disminución. La explicación de la disminución del volumen de plasma inducido por la hipoxia altitudinal no es fácil, pudiéndose argumentar desde procesos de redistribución de fluidos hasta las medidas, más sólidas, de valores menores en la concentración de aldosterona Whayne (2014) para habitantes de alturas mayores a 2.000 $\mathrm{m}$ o de la mayor concentración del factor natriurético atrial, comunicado por Schmidt et al. (1999) en controles y deportistas nativos de $2.000 \mathrm{~m}$ de altitud. Aldosterona y el factor natriurético atrial tienen acciones importantes sobre la regulación del volumen de agua en vertebrados. Un caso especial y hasta en cierta forma paradójico presentan los grupos de residentes, hombres y mujeres, entrenados físicamente en su altitud de $2.600 \mathrm{~m}$. Pomeroy y col (2015), midieron en ellos el volumen de plasma y de sangre, encontrando que los estímulos hipóxicos del entrenamiento no solamente reviertan el $8 \%$ de pérdida que se mencionó para grupos de no entrenados, sino que al igual de lo que sucede para entrenados a nivel del mar, hay una expansión de esos volúmenes en comparación con los no entrenados.
Así pues, en la bibliografía pertinente a la respuesta hemática para adaptación a la hipoxia altitudinal, se pueden encontrar resultados de hemoconcentración y de hemodilución que parecen responder más a los métodos de medida que lo estiman y poco al hecho o mecanismo fisiológico que pueda explicar la adaptación de dicha condición ambiental y geográfica

La respuesta hipóxica, sobre la que se dispone de nuevos datos críticamente importantes, puede esquematizarse en tres sistemas, por ejemplo de detección o sensor de oxígeno, de regulación, que controla la expresión génica y efector. El elemento principal de organización del sistema regulador es un factor de transcripción específico, el factor inducible por hipoxia 1 (HIF-1). En presencia de oxígeno, la subunidad $\alpha$ del HIF-1 (HIF-1 $\alpha$ ) se modifica por las hidroxilasas, que constituyen el punto central del mecanismo sensor, induciendo su catabolismo por el proteosoma. Por el contrario, en hipoxia, o en presencia de algunos factores de crecimiento que incrementan su síntesis, el HIF-1 $\alpha$ se transloca al núcleo, donde, unido al HIF-1 $\beta$, actúa como factor transcripcional de genes con elementos de respuesta hipóxica (HRE) en su promotor. Estos regulan la síntesis de una amplia serie de proteínas, que abarcan desde enzimas respiratorias y transportadores hasta hormonas involucradas en la regulación a escala del organismo de la circulación y la eritropoyesis. El papel del HIF-1 no se restringe a la mera inducción de una respuesta adaptativa a la falta de oxígeno, sino que participa significativamente en los mecanismos de reparación celular. Una simple lista de algunas alteraciones de importancia fisiopatológica, tanto estimulatorias como inhibitorias, que involucran al sistema de HIF-1, incluiría: enfermedad pulmonar crónica, adaptación al tabaco/humo, anemia/hemorragia, isquemia/reperfusión, crecimiento, vascularización y resistencia celular de los tumores, preeclampsia y crecimiento intrauterino retardado, hiper o hipovascularización retiniana, sobredosis de fármacos, enfermedad inflamatoria intestinal y curación de heridas. Vargas y Col (2006).

No se encontró diferencia significativa en el recuento leucocitario en los pobladores de Chivay, Yanque y Achoma $(p>0.05)$. El recuento de los leucocitos está dentro de los límites normales. Estos resultados coinciden con lo reportado por Hurtado 1972, Gonzales 2008. Demostraron que los leucocitos en los nativos de la altura no son diferentes a los observados a nivel del mar. Bighan y Col (2008) muestra para varones de Morococha (4540 m) que los granulocitos son similares que a nivel del mar. La fórmula leucocitaria es similar en nativos de nivel del mar y en la altura. Los leucocitos tampoco fueron diferentes en mujeres de nivel del mar y de la altura Whayne y col (2014) han estudiado parámetros de la inmunidad humoral en pobladores de altura. Para su realización se hicieron determinaciones de proteínas totales y fraccionadas por inmunoelectroforesis, cuantificación de inmunoglobulinas por inmunodifusión radial y detección de anticuerpos antinucleares en muestras provenientes de 44 personas normales, naturales y residentes de Cerro de Paseo (4,340 m). Los estudios demostraron que las concentraciones totales y fraccionadas de proteínas séricas estuvieron dentro de los valores considerados normales para habitantes del nivel del mar. Las cuantificaciones de inmunoglobulinas demostraron similitud con los del nivel del mar. Los anticuerpos antinucleares fueron negativos en 
la totalidad de las muestras. Los estudios parecen demostrar que los habitantes de las grandes alturas poseen parámetros inmunológicos humorales comparables a los del nivel del mar.

El número de leucocitos y de linfocitos $\mathrm{T}$ y $\mathrm{B}$ fueron similares a nivel del mar y en la altura Kiyamu y Col (2015). Durante el ejercicio se observa una leucocitosis que es considerada como normal. Hurtado (1964) demuestra que el nativo de la altura aumenta los leucocitos en un valor que resulta ser la mitad del observado a nivel del mar. No se sabe la implicancia fisiopatológica de este hallazgo. De acuerdo a Hurtado (1966), la leucemia es una entidad rara en los nativos de las grandes alturas.

Se encontró diferencia significativa en el VCM $(p<0.01)$. No se encontró diferencia significativa en los parámetros corpusculares HCM, CHCM. En la prueba de postcomparación de Tukey se encontró un aumento significativo $(\mathrm{p}<0.05)$ en el VCM de los individuos residentes de Chivay. . Los resultados de la masa de $\mathrm{Hb}$ en hombres y mujeres (estas últimas menores de 50 años de edad) residentes en altitud intermedia, $2.600 \mathrm{~m}$, no presentan igual magnitud. Los resultados obtenidos por Barbieri y col (2014), señalan que en los hombres (residentes a 2.600 $\mathrm{m})$, de todas las edades, la masa de Hb está incrementada en $12-28 \%$ con respecto a los del nivel del mar, el rango del incremento parece correlacionado con la respuesta a la hipoxia altitudinal, así como a su nivel de entrenamiento y exigencia del estímulo físico de cada deporte. Recientemente Benziger y Col (2015), comunican que para unos grupos de mujeres, menores de 50 años, no entrenadas, residentes de $40,1.000$ y $2.600 \mathrm{~m}$ de altitud, los valores de la masa de $\mathrm{Hb}$ ( $\mathrm{g} / \mathrm{Kg}$ peso corporal) no es diferente, a su vez las entrenadas, menores de 35 años, hacen un incremento de la masa de $\mathrm{Hb}$, pero este incremento es una respuesta al entrenamiento, no a la hipoxia altitudinal. Estos resultados son diferentes a lo medido para hombres, tal como ya se mencionó. Es probable pensar que la no respuesta eritropoyética medida en mujeres, menores de 50 años, esté explicada por el favorecimiento ventilatorio que en ellas hacen las hormonas femeninas, particularmente la progesterona. Resultados no publicados aún, de un trabajo realizado por nuestro grupo, muestran que en mujeres posmenopáusicas, la masa de $\mathrm{Hb}$ de residentes de la altitud está aumentada en forma significativa, 9 - $12 \%$, en relación con lo medido para las mujeres de la edad menor a 35 años, hecho que señala los efectos hormonales sobre este resultado. Los efectos ventilatorios mejorados en mujeres menores de 36 años también estarían reflejados en los valores, ya presentados, de la saturación de la hemoglobina, recuérdese que a 2.600 m.s.n.m., entre ese grupo de mujeres y los hombres, existe un $5 \%$ de más saturación en las mujeres, pero esta diferencia parece comenzar a perderse a partir de los 36 años de edad, aquí las mujeres tienen ya un $2 \%$ menos de saturación en la $\mathrm{Hb}$ que en los hombres y esta diferencia se amplía aún más después de los 50 años. El decremento ventilatorio, relacionado con el deterioro hormonal posmenopáusico parece ser la causa de esta última respuesta. Otra posibilidad que requiere de más investigación, es determinar si en las mujeres menores de 36 años los 2.600 m.s.n.m., no sean aún el umbral hipóxico a partir del cual se presente la respuesta eritropoyética compensatoria al descenso de la PIO2.

Es posible que la respuesta eritropoyética, compensatoria, representada por incrementos en la masa de $\mathrm{Hb}$, o en los valores de Hct, no sea más que el resultado final de órdenes genéticas sobreexpresadas. Un artículo de Pawson y Col (2010), comunica resultados que permiten clasificar a personas como respondedoras y a otras no respondedoras a los estímulos hipóxicos. La clasificación se basa en los incrementos de valores diferentes de la eritropoyetina en personas expuestas en forma aguda a una altura mayor a los 200 m.s.n.m., los de respuesta incrementada serían los de mejor adaptación. Aun cuando con resultados contradictorios, otros estudios como los de Brutsaert y col (2003), indican que algunas mutaciones o polimorfismos encontrados para genes de la enzima convertidora de angiotensina, ACE, y del fibrinógeno pueden favorecer la adaptación a la hipoxia altitudinal, la primera estaría promoviendo adaptaciones desde el volumen plasmático y la segunda desde la característica física de la menor viscosidad de la sangre. Un mayor volumen plasmático puede propiciar una mejora en el gasto cardiaco y una sangre menos viscosa presentaría una difusión de oxígeno con menos resistencia que la que se encontraría en un fluido de gran viscosidad.

Se encontró diferencia significativa en el pulso $(p<0.05)$. No se encontró diferencia significativa en los parámetros corpusculares PS, PD, PP, PMF. En la prueba de postcomparación de Tukey se encontró un aumento significativo $(p<0.05)$ en el pulso de los individuos residentes de Chivay. Es conocido desde antes que la prevalencia de Hipertensión arterial (HTA) y arterioesclerosis es menor en la altura. Esto es cierto para la presión sistólica mas no así para la diastólica que como se sabe depende de la viscosidad sanguínea. Benziger y Col (2015), han observado que las personas con eritrocitosis excesiva (nivel de hemoglobina más de dos desviaciones estándar de la media) están más predispuesta a tener hipertensión diastólica que los que tienen eritrocitosis fisiológica. La reducción de la presión sistólica ha sido atribuida a una menor resistencia periférica, ocasionado por un incremento de la vascularización vasodilatación, mecanismos adaptativos orientados a mejorar el aporte sanguíneo de oxígeno a los tejidos. Al parecer la menor prevalencia de HTA observada es al menor grado de arterioesclerosis.

No se encontró diferencia significativa en el diámetro y frecuencia respiratoria $(p>0.05)$. Estos valores comparados con los del nivel del mar son mayores. Estos resultados coinciden con lo reportado por Vargas y Col (2006) quienes reportan que uno de los parámetros de climatización del ser humano a la altura y su posterior adaptación produce cambios en la anatomía, como el ensanchamiento del tórax. A la disminución de la presión parcial de oxígeno, se muestra un cambio a nivel del torax más amplio en sentido vertical, transversal y anteroposterior, un aspecto que, por lo demás, ha sido comprobado a nivel antropológico y morfométrico

Cuando un individuo que normalmente habita a nivel del mar asciende a la altura sufre una serie de mecanismos de aclimatización como aumento de la frecuencia respiratoria en forma transitoria, aumento en días sucesivos del nivel de hemoglobina y hematocrito, esto debido a la disminución de la presión parcial de oxígeno que estimula a los quimiorreceptores localizados en la bifurcación de la carótida y el cayado aórtico. La hipoxemia también produce un aumento transitorio de la eritropoyetina que a su vez produce eritrocitosis fisiológica. En la exposición aguda hay 
un aumento de la ventilación alveolar, la $\mathrm{PaCO} 2$ disminuida estimula la excreción de bicarbonato sanguíneo por los riñones y se restaura el $\mathrm{pH}$ normal o casi normal de modo que la alcalosis respiratoria se compensa. En el habitante de altura hay una disminución del gradiente alvéoloarterial que a nivel del mar es de $10 \mathrm{mmHg}$ y Morococha (4500 m.s.n.m) es de casi cero. Está demostrado que la capacidad de difusión de la membrana alveoloarterial está aumentado en la altura. Este aumento depende de un incremento en el tamaño de las membranas, es decir aumento en el área de difusión, más capilares gruesos y alvéolos dilatados Kiyamu y col (2015)..

La cantidad de sangre que llevan los vasos pulmonares es mucho mayor en la altura. En Lima el 15\% del volumen total de sangre $(4,8 \mathrm{~L})$ está en los pulmones, mientras que en Morococha el $20 \%$ del volumen total de sangre $(5,7$ L.) están en el pulmón. Es decir $720 \mathrm{ml}$ contra $1100 \mathrm{ml}$. Esta mayor cantidad de líquido hace que la elasticidad disminuya con el consiguiente efecto mecánico: dilatación del tórax. La capacidad vital y el volumen residual está aumentados en el habitante de altura. Lo anterior se debe a que existe un aumento en el diámetro antero posterior del tórax Reeves y $\operatorname{col}(1985)$

La respiración de los nativos de tierras muy altas responde menos a la hipoxia, de modo que siempre tienen una ventilación disminuida a alturas mayores, en comparación con las personas de la misma raza que viven a nivel del mar y ascienden transitoriamente a las mismas elevaciones. En estos nativos de grandes alturas los grados de hipoxia adicionales solo estimulan en forma mínima el impulso ventilatorio. Esta respuesta sería genética o se adquiriría a temprana edad como respuesta del ambiente De Ferrari y Col (2014).

La cantidad de hemoglobina que se une a la sangre es proporcional a la presión del 02 , pero la relación entre la $\mathrm{Hb} 02$ y la Pao2 no es lineal sino exponencial y la curva que lo representa se denomina curva de disociación de la $\mathrm{Hb}$. En la altura hay una desviación a la derecha de esta gráfica Lindgerde y col (2004).

\section{REFERENCIAS BIBLIOGRÁFICAS}

1. ANDAGUA,R.; RAMOS,J. 1967 Variaciones leucocitarias en la exposición aguda a las grandes alturas. Arch. Inst. Biol. Andina Vol 2 pág 75

2. BARBIERI C, HEGGARTY P, YANG YAO D, FERRI G, DE FANTI S, SARNO S, CIANI G, BOATTINI A, LUISELLI D, PETTENER D.Between Andes and Amazon: the genetic profile of the Arawak-speaking Yanesha. Am J Phys Anthropol. 2014 Dec;155(4):6009.

3. BENZIGER CP, BERNABÉ-ORTIZ A, GILMAN RH, CHECKLEYW, SMEETH L, MÁLAGAG, MIRANDA JJ Metabolic Abnormalities Are Common among South American Hispanics Subjects with Normal Weight or Excess Body Weight: The CRONICAS Cohort Study. PLoS One. 2015 Nov 23;10(11):e0138968.

4. BIGHAM AW, KIYAMU M, LEÓN-VELARDE F, PARRA EJ, RIVERA-CH M, SHRIVER MD, BRUTSAERT TD. Angiotensin-converting enzyme genotype and arterial oxygen saturation at high altitude in Peruvian Quechua. High Alt Med Biol. 2008 Summer;9(2):167-78.

5. BONNING,D.;MAASEN,N.; JOCKUN,F. 1997 After efects of a high altitude expedition on blood. INST. J
SPORT MEDICINE. April 18(3).pág. 179-85

6. BRUTSAERT TD, PARRA EJ, SHRIVER MD, GAMBOA A, PALACIOS JA, RIVERA M, RODRIGUEZ I, LEÓN-VELARDE F. Spanish genetic admixture is associated with larger $\mathrm{V}(\mathrm{O} 2)$ max decrement from sea level to $4338 \mathrm{~m}$ in Peruvian Quechua. J Appl Physiol (1985). 2003 Aug;95(2):51928.

7. CHARA,B.C. 1993 Valores hematológicos en sangre periférica y hematogenesis de ratas sometidas a 80,2290 Y $2964 \mathrm{msnm}$. Tesis para optar el título profesional de Biólogo U.N.S.A.

8. COUDERT,J.; PAZ,Z.M.,ANTEZANA,G. Y VARGAS,E.; 1971 Valores hemodinámicos normales de la altura, LA PAZ 3700 msnm. Arch. Inst. Biol. Andina 4(2,3 y 4): pág 94-97 Mayo/Diciembre

9. DE FERRARI A, MIRANDA JJ, GILMAN RH, DÁVILA-ROMÁN VG, LEÓN-VELARDE F, RIVERA-CH M, HUICHO L, BERNABÉ-ORTIZ A, WISE RA, CHECKLEY W Prevalence, clinical profile, iron status, and subject-specific traits for excessive erythrocytosis in andean adults living permanently at 3,825 meters above sea level. Chest. 2014 Nov;146(5):1327-36.

10. DE LA CRUZ MG, MALPARTIDA SB, SANTIAGO HB, JULLIAN V, BOURDY G. Hot and cold: medicinal plant uses in Quechua speaking communities in the high Andes (Callejón de Huaylas, Ancash, Perú). J Ethnopharmacol. 2014 Sep 11;155(2):1093-117.

11. FAVIER,R..; SPIELVOGEL,H.; DESPLANCHES,D.; FERRETI,G. 1995 Training in hipoxia vs training in normoxia in high altitude natives. J.Appl.physiol.Jun 78(6) pág 2286-93.

12. FRISANCHO,A.R. 1979 Human adaptation a functional interpretation THE C.V. Mosby Company Missouri.

13. FRISANCHO,A.R., $\quad$ FRISANCHO,H.G.; MILOTICH,M.; $\quad$ BRUTSAERT,T.; 1995 Developmental genetic and envirmental components of aerobic capacity at high altitude. Am.J. Phys Antropol April 96(4) pág 431-42

14. GAMBOA,R. 1998 Fisiología y fisiopatología cardiovascular durante la exposición aguda a la altura. Acta Andina 7(1) pág 35-46

15. GANONG,W.F. 1988 Fisiología Médica $11^{\mathrm{a}}$ Edición. Editorial El Manual Moderno S.A. de CV.México D.F.

16. GOMEZ,C.E.; 1990 Valores de hemoglobina, hematocrito y poliglobulia de altura en la poblacion que atiende el HOSPITAL DE APOYO II IPSS DE JULIACA. Tesis para optar el grado de Bachiller en Medicina Humana. U.N.S.A.

17. GUYTON,A.C.1986 Tratado de Fisiología Médica. 7 ma Edición. Editorial Interamericana Mc.Graw Hill México.

18. HASLER,E; SUTTER,P.M.; VETTER,W.; 1997 Race specific altitude effects on blood pressure. J. Hypertenses 11(7) pág 435-8

19. HEATH,D.; WILLIAMS,D.R. 1981 Man at high altitude. the pathophysiology of aclimatization and adaptation. Second Edition.

20. HOFF,CH.; BAKER,P.T. ; GARRUTO,R.; 1972 Variaciones altitudinales en pulso y presion arterial en 
nativos andinos del Perú. Arch. Inst. Biol. Andina 5(2) pág 57-67 Julio/Diciembre.

21. HURTADO,A. 1978 Aspectos fisiológicos y patológicos de la vida en la altura. Acta Médica Peruana Vol V 15 pág 28-34

22. KIYAMU M, BIGHAM A, PARRA E, LEÓNVELARDE F, RIVERA-CHIRA M, BRUTSAERT TD.Developmental and genetic components explain enhanced pulmonary volumes of female Peruvian Quechua. Am J Phys Anthropol. 2012 Aug;148(4):53442.

23. KIYAMU M, LEÓN-VELARDE F, RIVERA-CHIRA $\mathrm{M}$, ELÍAS G, BRUTSAERT TD Developmental Effects Determine Submaximal Arterial Oxygen Saturation in Peruvian Quechua. High Alt Med Biol. 2015 Jun;16(2):138-46.

24. KIYAMU M, RIVERA-CHIRA M, BRUTSAERT TD Aerobic capacity of Peruvian Quechua: a test of the developmental adaptation hypothesis. Am J Phys Anthropol. 2015 Mar;156(3):363-73.

25. LAZO,V.J.O-; HURTADO,O.E.H. 1989 Presion alterial en adultos supuestamente sanos de 20-44 años de edad a 3827 metros de altura en Puno. Tesis para optar el grado de Bachiller en Medicina Humana UNSA.

26. LEON,V.G., ARREGUI,A. 1994 Desadaptación a la vida en las grandes alturas. Instituto Francés de Estudios Andinos (IFEA).

27. LINDGÄRDE F, ERCILLA MB, CORREA LR, AHRÉN B. Body adiposity, insulin, and leptin in subgroups of Peruvian Amerindians. High Alt Med Biol. 2004 Spring;5(1):27-31.

28. MEYER,M.; RAHNEL,A.; MARCONI,C. 1999 Stability of heart beat interval distributions in chronic high altitude hipoxia. Integr. Physiol. Behay.Sci. October-December 33(4) pág 344-62

29. PALACIOS,M.S.B.; 1995 algunos valores hematológicos y séricos normales en los pobladores del colca, Coporaque. Tesis para optar el grado de Bachiller en Medicina Humana U.N.S.A.

30. PAWSON IG, HUICHO L.Persistence of growth stunting in a Peruvian high altitude community, 19641999. Am J Hum Biol. 2010 May-Jun;22(3):367-74.

31. PIEDRAS,J.;LORIA,A.; GALVAN,I. 1995 Red blood indices in high altitude hospital population. Arch. Med Resid. Spring 26(1) pág 65-68

32. POMEROY E, WELLS JC, STANOJEVIC S, MIRANDA JJ, MOORE LG, COLE TJ, STOCK JT Surname-inferred Andean ancestry is associated with child stature and limb lengths at high altitude in Peru, but not at sea level. Am J Hum Biol. 2015 NovDec;27(6):798-806

33. PORTER,R ;; KNIGHT,J.; 1971 High altitude physiology, cardiac and respiratory aspects. Edit. Churchill Livinstone London

34. REEVES JT, GROVER RF. Insights by Peruvian scientists into the pathogenesis of human chronic hypoxic pulmonary hypertension. J Appl Physiol (1985). 2005 Jan;98(1):384-9.

35. RUPERT JL, KIDD KK, NORMAN LE, MONSALVE MV, HOCHACHKA PW, DEVINE DV. Genetic polymorphisms in the Renin-Angiotensin system in high-altitude and low-altitude Native American populations. Ann Hum Genet. 2003 Jan;67(Pt 1):17-25.

36. SAUVEREY,G.; GARCIA,N.; BESNARD,Y.1996 Pre -adaptation and de -adaptation to high altitude in humans: cardio ventilatory an hematological changes . Eur. J. Appl. Physiol. 73(6) pág 529-35

37. SIME,B.F.J.; 1973 Ventilación humana en hipoxia crónica, etiopatogenia de la enfermedad de Monge o desadaptación crónica a la altura. Tesis Doctoral presentado en la Universidad Peruana Cayetano Heredia.

38. SUCA,Y.J. 1974 Valores normales de algunos aspectos somatométricos y fisiológicos en trabajadores a nivel del mar y a 3000 metros de altura. Tesis para obtar el grado de Bachiller en Medicina Humana U.N.S.A.

39. VARGAS E, SPIELVOGEL H. Chronic mountain sickness, optimal hemoglobin, and heart disease. High Alt Med Biol. 2006 Summer;7(2):138-49. 